\title{
Numerical investigation on detonation cell evolution in a channel with area-changing cross section
}

\author{
DENG Bo ${ }^{\dagger}$, HU ZongMin, TENG HongHui \& JIANG ZongLin \\ Key Laboratory of High Temperature Gas Dynamics, Institute of Mechanics, Chinese Academy of Sciences, \\ Beijing 100080, China
}

The two-dimensional cellular detonation propagating in a channel with areachanging cross section was numerically simulated with the dispersion-controlled dissipative scheme and a detailed chemical reaction model. Effects of the flow expansion and compression on the cellular detonation cell were investigated to illustrate the mechanism of the transverse wave development and the cellular detonation cell evolution. By examining gas composition variations behind the leading shock, the chemical reaction rate, the reaction zone length, and thermodynamic parameters, two kinds of the abnormal detonation waves were identified. To explore their development mechanism, chemical reactions, reflected shocks and rarefaction waves were discussed, which interact with each other and affect the cellular detonation in different ways.

detonation cells, transverse waves, detailed chemical reaction model, numerical simulation

\section{Introduction}

The detonation wave with complicated wave structures is one kind of supersonic combustion. Many studies from different viewpoints were carried out to explore its thermodynamic parameters, dynamic scales, wave front structures and cellular detonation cells. By neglecting the wave structure and chemical reactions, the C-J theory was proposed to predict the thermodynamic parameters with reasonable accuracy, such as the detonation speed, the post-wave pressure and temperature. The ZND model ${ }^{[1]}$ was developed by modifying the C-J theory to introduce the induction and heat release zones to mimic the chemical reactions. Although the ZND detonation structure is different from the experimental observations, it initiated the new research by considering both thermodynamic parameters and hydrodynamic scales together, which shows the promising direction of the future experimental and numerical investigations on detonation dynamic scales, wave front structures and cellular detonation cells ${ }^{[2,3]}$. Further study showed that the detonation front is of the three-dimensional structure that consists of incident shocks, Mach stems,

Received April 10, 2006; accepted January 8, 2007

doi: 10.1007/s11433-007-0076-0

${ }^{\dagger}$ Corresponding author (email: dengbo@imech.ac.cn) 
transverse waves, reaction zones and shear layers. This structure was demonstrated by the periodic cellular detonation cells recorded by using smoked foil technology. Pioneer research work also showed that the front structure and the cellular detonation cells mainly depend on the gaseous mixture composition and its initial states, and indicate the interaction between chemical reactions and shock waves. Further investigation into the cellular detonation patterns is vital in exploring the nature of various detonation phenomena.

When detonation waves propagate in a channel with area-changing cross section, the wave front structure and cellular detonation cells may vary due to the flow expansion or compression. Jones et al. ${ }^{[4]}$ investigated the detonation quenching and re-igniting in wave diffraction, and demonstrated that there are two kinds of detonation initiation mechanisms: the collision between the triple points and the inducement of high temperature reaction zones. Guo et al. ${ }^{[5]}$ experimentally visualized the detonation reflection from wedges and observed much smaller detonation cells behind Mach stems by using smoked foil record technology. Thomas et al. ${ }^{[6]}$ reported a large-scale experiment on the detonation propagation in the mixture of acetylene-oxygen at various divergence wall divergence angles, and demonstrated that as the wall angle increases, the size of detonation cells near the wall becomes larger and larger. However, the above-mentioned work revealed the important detonation phenomena, but the nature of detonation cell evolution has not been clearly interpreted, yet. Furthermore, if the detonation front structure depends only on the mixture composition and its initial states stand for the normal detonation, what are the major differences between the normal detonations and the detonations having different cell size due to the post-shock state change for the same gas mixture and initial conditions?

The two-dimensional cellular detonation propagating in a channel with area-changing cross section was numerically simulated with the dispersion controlled dissipative scheme (DCD) and the detailed chemical reaction kinetics. The scheme was applied successfully in detonation simulation with the one-step reaction models ${ }^{[7,8]}$, and the further extension was made to investigate effects of flow expansion and compression on cellular detonation cell to gain better understanding of the interaction among the reaction zone, the chemical reaction rate and the wave front structure.

\section{Governing equations and the numerical method}

\subsection{Governing equations}

Assuming that dissipative effects, such as viscous, heat-conductive and diffusive effects, are neglected for the study on detonation propagation, the governing equations of gaseous detonations are two-dimensional multi-component Euler equations with chemical reaction source terms. The equations can be written in the conservation form with $n s$ continuity equations for a perfect gaseous mixture in Cartesian coordinates:

$$
\begin{gathered}
\frac{\partial \boldsymbol{U}}{\partial t}+\frac{\partial \boldsymbol{F}}{\partial x}+\frac{\partial \boldsymbol{G}}{\partial y}=\boldsymbol{S}, \\
\boldsymbol{U}=\left[\begin{array}{c}
\rho C_{1} \\
\rho C_{2} \\
\vdots \\
\rho C_{n s} \\
m \\
n \\
E
\end{array}\right], \quad \boldsymbol{F}=\left[\begin{array}{c}
m C_{1} \\
m C_{2} \\
\vdots \\
m C_{n s} \\
m^{2} / \rho+p \\
m n / \rho \\
(E+p) m / \rho
\end{array}\right], \quad \boldsymbol{G}=\left[\begin{array}{c}
n C_{1} \\
n C_{2} \\
\vdots \\
n C_{n s} \\
m n / \rho \\
n^{2} / \rho+p \\
(E+p) n / p
\end{array}\right], \quad \boldsymbol{S}=\left[\begin{array}{c}
\dot{\omega}_{1} \\
\dot{\omega}_{2} \\
\vdots \\
\dot{\omega}_{n s} \\
0 \\
0 \\
0
\end{array}\right],
\end{gathered}
$$


where $\boldsymbol{U}$ is an unknown variable, $\boldsymbol{F}$ and $\boldsymbol{G}$ the numerical flux, $\boldsymbol{S}$ the chemical reaction terms. $c_{i}=\rho_{i} / \rho(i=1, n s)$ indicates mass fraction of the species $i$ and $\rho_{i}$ is its density. $m=\rho u$, $n=\rho v$, and $u$ and $v$ are velocity component in $x$ - and $y$-direction, respectively. The total specific energy is defined as $E=\rho h-p+\rho v^{2} / 2$ and it can be expressed as $E=e+\rho\left(u^{2}+v^{2}\right) / 2$ with the internal specific energy being $e=\rho h-p$. According to Dalton's law, the flow pressure $p$ is the sum of the partial pressure for each species, and can be calculated with the equation of state for a perfect gas, $p=\sum_{i=1}^{n_{s}} \rho_{i} R_{i} T$, where $R_{i}$ is the gas constant of species $i$, and $T$ the temperature of the gaseous mixture.

To simulate the detonation propagation process in the hydrogen and oxygen mixture, the 8 species $\left(\mathrm{H}_{2}, \mathrm{O}_{2}, \mathrm{O}, \mathrm{H}, \mathrm{OH}, \mathrm{HO}_{2}, \mathrm{H}_{2} \mathrm{O}_{2}, \mathrm{H}_{2} \mathrm{O}\right)$ detailed chemical reaction model including 20 reactions is adopted. The general reaction formula is written in the form of

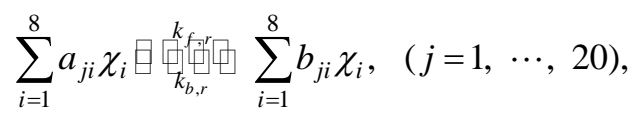

where the molecular concentration of each species is denoted by $\chi_{j} ; k_{f, r}$ and $k_{b, r}$ denote the forward and the backward reaction rates, respectively. For elementary chemical reactions, the chemical reaction rates $\dot{\omega}_{i}$, derived from a reaction mechanism consisting of NR chemical reactions, can be calculated by

$$
\dot{\omega}_{i}=w_{i} \sum_{r=1}^{N R}\left(v_{i r}^{\prime}-v_{i r}^{\prime \prime}\right)\left(k_{f, r} \prod_{j=1}^{n_{s}}\left(\chi_{j}\right)^{v_{i r}^{\prime}}-k_{b, r} \prod_{j=1}^{n_{s}}\left(\chi_{j}\right)^{v_{i r}^{\prime \prime}}\right),
$$

where $i$ and $j$ denote the species in the $r$ th elementary reaction; the molecular weight of each species is denoted by $w_{i} ; v_{i r}^{\prime}$ and $v_{i r}^{\prime \prime}$ are stoichiometric coefficients of species $i$, appearing as a reactant and a product. The forward reaction rate of each reaction is calculated with the Arrhenius law, and the corresponding backward reaction rate can be derived from the equilibrium constant and $k_{f, r}$.

\subsection{Numerical method}

For numerical simulations, the second-order explicit difference equations of eq. (1) discretized both in space and time by using the dispersion-controlled dissipative scheme $e^{[9,10]}$ proposed by Jiang et al. for shock wave capturing. The time-marching integration was performed by using a Runge-Kutta algorithm of second-order accuracy:

$$
\boldsymbol{U}_{j, k}^{n+1}=\boldsymbol{U}_{j, k}^{n}-\frac{\Delta t}{\Delta x}\left(\overline{\boldsymbol{F}}_{j+1 / 2, k}^{n}-\overline{\boldsymbol{F}}_{j-1 / 2, k}^{n}\right)-\frac{\Delta t}{\Delta y}\left(\overline{\boldsymbol{G}}_{j, k+1 / 2}^{n}-\overline{\boldsymbol{G}}_{j, k-1 / 2}^{n}\right)-\frac{\Delta t}{y} \boldsymbol{S}_{g j, k}^{n}-\Delta t \boldsymbol{S}_{j, k}^{n},
$$

here,

$$
\begin{gathered}
\left\{\begin{array}{l}
\overline{\boldsymbol{F}}_{j+1 / 2}^{n}=\boldsymbol{F}_{j+1 / 2 L, k}^{+}+\boldsymbol{F}_{j-1 / 2 R, k}^{-}, \\
\overline{\boldsymbol{G}}_{j, k+1 / 2}^{n}=\boldsymbol{G}_{j, k+1 / 2 L}^{+}+\boldsymbol{G}_{j, k-1 / 2 R}^{-},
\end{array}\right. \\
\left\{\begin{array}{l}
\boldsymbol{F}_{j+1 / 2 L, k}^{+}=\boldsymbol{F}_{j, k}^{+}+\frac{1}{2} \boldsymbol{\Phi}_{A}^{+} \min \bmod \left(\Delta \boldsymbol{F}_{j-1 / 2, k}^{+}, \Delta \boldsymbol{F}_{j+1 / 2, k}^{+}\right), \\
\boldsymbol{F}_{j+1 / 2 R, k}^{-}=\boldsymbol{F}_{j+1, k}^{-}-\frac{1}{2} \boldsymbol{\Phi}_{A}^{-} \min \bmod \left(\Delta \boldsymbol{F}_{j+1 / 2, k}^{-}, \Delta \boldsymbol{F}_{j+3 / 2, k}^{-}\right),
\end{array}\right.
\end{gathered}
$$




$$
\begin{aligned}
& \left\{\begin{array}{l}
\boldsymbol{G}_{j, k+1 / 2 L}^{+}=\boldsymbol{G}_{j, k}^{+}+\frac{1}{2} \boldsymbol{\Phi}_{B}^{+} \min \bmod \left(\Delta \boldsymbol{G}_{j, k-1 / 2}^{+}, \Delta \boldsymbol{G}_{j, k+1 / 2}^{+}\right), \\
\boldsymbol{G}_{j, k+1 / 2 R}^{-}=\boldsymbol{G}_{j, k+1}^{-}-\frac{1}{2} \boldsymbol{\Phi}_{B}^{-} \min \bmod \left(\Delta \boldsymbol{G}_{j, k+1 / 2}^{-}, \Delta \boldsymbol{G}_{j, k+3 / 2}^{-}\right),
\end{array}\right. \\
& \left\{\begin{array}{l}
\Delta \boldsymbol{F}_{j+1 / 2, k}^{ \pm}=\boldsymbol{F}_{j+1, k}^{ \pm}-\boldsymbol{F}_{j, k}^{ \pm}, \\
\Delta \boldsymbol{G}_{j, k+1 / 2}^{ \pm}=\boldsymbol{G}_{j, k+1}^{ \pm}-\boldsymbol{G}_{j, k}^{ \pm},
\end{array}\right. \\
& \left\{\begin{array}{l}
\boldsymbol{F}^{ \pm}=\boldsymbol{A}^{ \pm} \boldsymbol{U}, \\
\boldsymbol{G}^{ \pm}=\boldsymbol{B}^{ \pm} \boldsymbol{U},
\end{array}\right. \\
& \left\{\begin{array}{l}
\boldsymbol{\Phi}_{A}^{ \pm}=\boldsymbol{I} \mp \beta \boldsymbol{\Lambda}_{A}^{ \pm}, \\
\boldsymbol{\Phi}_{B}^{ \pm}=\boldsymbol{I} \mp \beta \boldsymbol{\Lambda}_{B}^{ \pm} .
\end{array}\right.
\end{aligned}
$$

In the above equations, $\boldsymbol{A}=\partial \boldsymbol{F} / \partial \boldsymbol{U}, \boldsymbol{B}=\partial \boldsymbol{G} / \partial \boldsymbol{U}, \quad I$ is unit matrix, $\beta=\Delta t / \Delta r$, and $\boldsymbol{\Lambda}_{A}$ and $\boldsymbol{\Lambda}_{B}$ are the matrixes consisting of the eigenvalues of matrix $\boldsymbol{A}$ and $\boldsymbol{B}$. In these equations, the $(\square)^{+}$and $(\square)^{-}$ superscripts denote flux vector splitting according to the Steger and Warming method. This scheme implemented with the multi-species detailed reaction model ${ }^{[11]}$ has been successfully applied in numerical simulations of detonations. In the detonation simulation, the stiff problem, which derives from the huge difference of the chemical and hydrodynamic time scales, occurs and is dealt with the splitting method, that is, the frozen flow field is simulated first, and then the chemical reaction influence is considered as a constant volume combustion process, which will change the densities, pressure and temperature. By doing so, the flow field and chemical reactions can be computed by using different time steps with a required accuracy.

\subsection{Numerical simulation verification}

To verify the applied numerical code and the chemical reaction model, the detonation reflecting from two wedges with different angles in a stoichiometric mixture of hydrogen and oxygen, diluted by $25 \%$ Argon, was calculated and compared with experimental schlierens as shown in Figure 1. Figure 1(a) shows the Mach reflection from a $30^{\circ}$ wedge angle and Figure 1(b) shows the regular reflection from a $60^{\circ}$ wedge angle. For each figure, the experimental result is presented in the upper half and the numerical one is plotted in the lower half. Both figures indicate that the angle of triple point trajectory, $\chi$, and detonation reflection patterns in both experiments and CFD are consistent with each other.

\section{Numerical results and discussion}

\subsection{Detonation cell and chemical reaction scale}

The detonation cellular structure evolution can be used to identify detonation front structure variations, self-sustained propagation, and even the detonation initiation and failing. Theoretical study showed that the detonation cell size and the degree of cell regularity mainly depend on the parameter, $\hat{E} a=E a / R T$, of the detonable mixtures, where $E a$ is the activation energy, $R$ is the universal gas constant, and $T$ is defined as the post-shock temperature in the ZND model. This characteristic parameter governs the chemical ignition delay time. Generally speaking, each species involving chemical reactions has it own activation energy $E a$, and the intensive variation of 

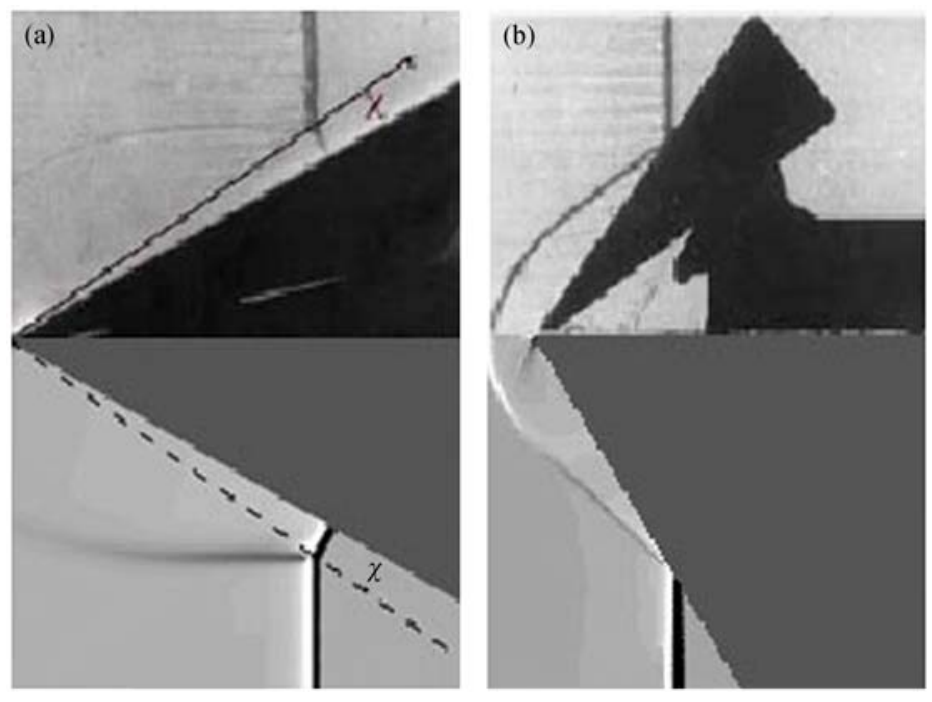

Figure 1 Comparison between numerical and experimental results of detonation reflection over wedges with numerical results shown in the lower half and experimental ones in the upper half. (a) A $30^{\circ}$ wedge angle; (b) a $60^{\circ}$ wedge angle.

the local shock velocity, triple point collisions and the un-reacted gas pockets may lead to the dramatically-changing temperature. Therefore, the resultant characteristic parameters of each species will affect the detonation cell size more or less, and therefore, the total characteristic parameter including all the effects of each species in chemical reactions is the key factor in determining the detonation cell size. Numerical simulations show that increasing $\hat{E} a$ of the detonable gas mixture favors the more irregular structure characterized by a high frequency of appearance and disappearance of the triple points.

The distribution of three normalized species $\left(2 \mathrm{H}_{2}+\mathrm{O}_{2}+\mathrm{Ar}, \mathrm{P}_{0}=16 \mathrm{kPa}\right)$ densities in onedimensional detonation is shown in Figure 2. It can be observed that the density distributions of $\mathrm{H}_{2}$, $\mathrm{H}$ and $\mathrm{OH}$ behind the leading shock vary in different tendency: $\mathrm{H}_{2}$ reaches its peak value at the reaction front because of shock wave compression, and then falls rapidly due to chemical reaction consuming; $\mathrm{H}$ and $\mathrm{OH}$ are produced considerably in the reaction zone, but $\mathrm{H}$ falls faster than $\mathrm{OH}$. This figure demonstrates that there are various length scales for reaction species, and each plays its different role in the detonation reaction system. Considering the idealized single-step or two-step reaction models where the realistic chemical reaction kinetics is over-simplified, it is believed that a detailed chemical reaction model can be more trustable to produce high qualitative results in the detonation cell simulation because the different chemical reaction length scales were modeled properly. The dynamic parameter used for characterization of the cellular detonation is the cell width $\lambda$. It was shown that the cell size $\lambda$ is related to the mean chemical induction length and the local detonation speed ${ }^{[12]}$. Desbordes reported that $\lambda$ is not only a dynamic parameter of the self-sustained Chapman-Jouguet detonation, but applicable to any detonation waves. If the detonation speed $D$ is higher than $D_{\mathrm{CJ}}$ of the Chapman-Jouguet detonation, the cell width diminishes as the detonation speed increases. This is because that the induction length of the chemical reaction zone behind the incident shock wave diminishes correspondingly. He showed that the correlation between the cell width $\lambda$ and the reaction induction length $L$ or detonation speed $D$ could be predicted analytically by the formula of 


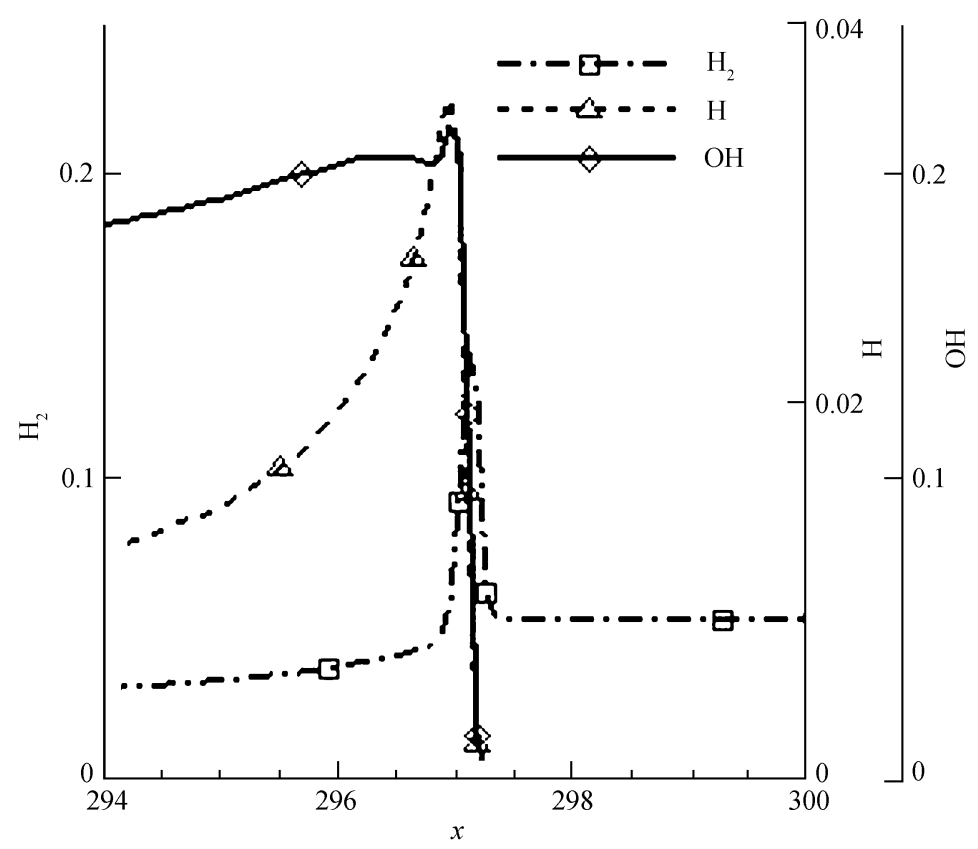

Figure 2 Variation of the normalized density of species $\left(2 \mathrm{H}_{2}+\mathrm{O}_{2}+\mathrm{Ar}, \mathrm{P}_{0}=16 \mathrm{kPa}\right)$ in one-dimensional detonation waves.

$$
\frac{\lambda}{\lambda_{\mathrm{CJ}}}=\frac{L_{\text {ind }}}{L_{\text {ind,CJ }}}=\frac{D}{D_{\text {CJ }}} \mathrm{e}^{\frac{E_{a}}{R T_{\text {ZND }}}\left[\left(\frac{D_{\mathrm{CJ}}}{D}\right)-1\right]},
$$

where $\lambda$ and $\lambda_{\mathrm{CJ}}$ are the cell widths, $L_{\text {ind }}$ and $L_{\text {ind,CJ }}$ denote the global chemical induction lengths, $D$ and $D_{\mathrm{CJ}}$ the detonation speed, respectively. The subscript, CJ, indicates Chapman-Jouguet (CJ) detonations. This is understandable that reaction length scales affect the cell generation and evolution. So, the pioneering numerical simulation in which only one chemical reaction length scale is considered has its limitation in the detonation cell study.

Figure 3 shows the evolution of a two-dimensional cellular detonation in the gaseous mixture of $2 \mathrm{H}_{2}+\mathrm{O}_{2}+$ Ar with $P_{0}=16 \mathrm{kPa}$. After artificial perturbations were induced, self-sustained detonation waves developed and periodical detonation cells were generated. Due to eliminating other kinds of perturbations, this typical numerical detonation has more advantages in the analysis of the cellular detonation evolution resulting from flow compression or expansion.

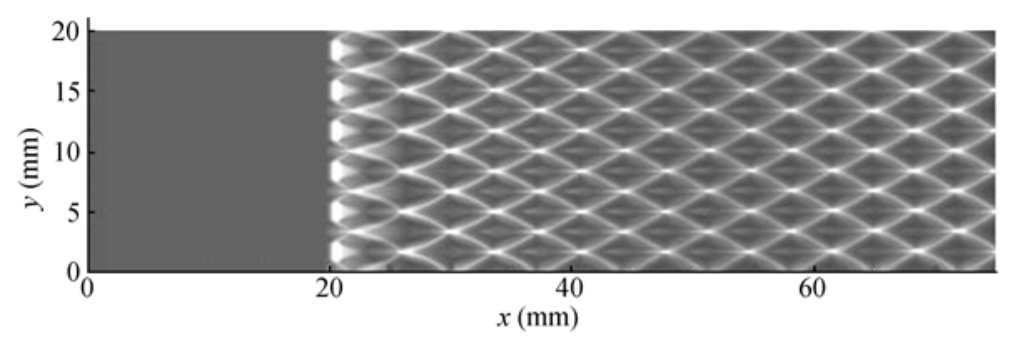

Figure 3 Numerical schlieren image of cellular detonation cell’s development after introducing artificial perturbations.

\subsection{Detonation cells in a converging channel}

Cellular detonation cells pattern resulting from the detonation propagating in a planar channel with 
a $20^{\circ}$ converging wedge is shown in Figure 4. Obviously, the cellular detonation front disassembles into two parts, the incident detonation and the Mach stem detonation which appears to be an overdriven one. The two detonations joint at the main triple point from which the trajectory develops due to the interaction between the incident detonation and the reflected shock wave. On each side of the trajectory, the cellular detonation cells pattern has a different cell size. The cell pattern behind the incident detonation wave remains unchanged, and the one behind the Mach stem becomes smaller, which indicates an overdriven detonation.

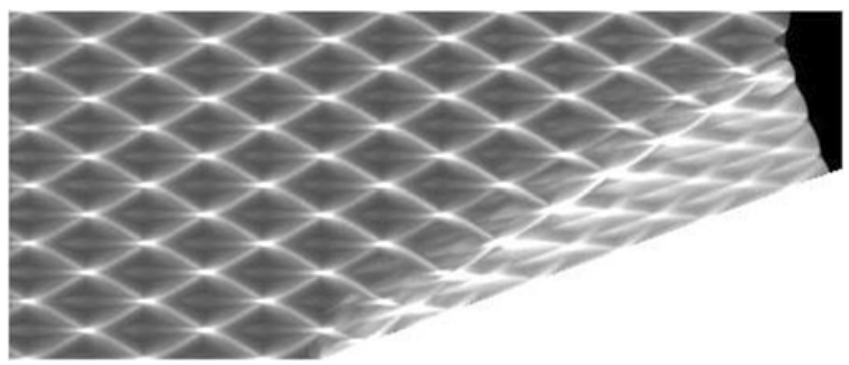

Figure 4 Cellular detonation cells pattern of detonation propagating in a planar channel with a $20^{\circ}$ converging wedge.

Figure 5 shows the successive post-shock pressure profiles during detonation propagating along the centerline (marked by the dot-dash line in the subfigure) of three typical cells (labeled as Cell-1, Cell-2, and Cell-3). Four peaks are observable in the pressure profiles due to triple-point collisions. Cell-1 locates outside the region swept by the Mach stem and the first peak is generated by the triple point collision in the incident detonation without any disturbance from detonation reflection.

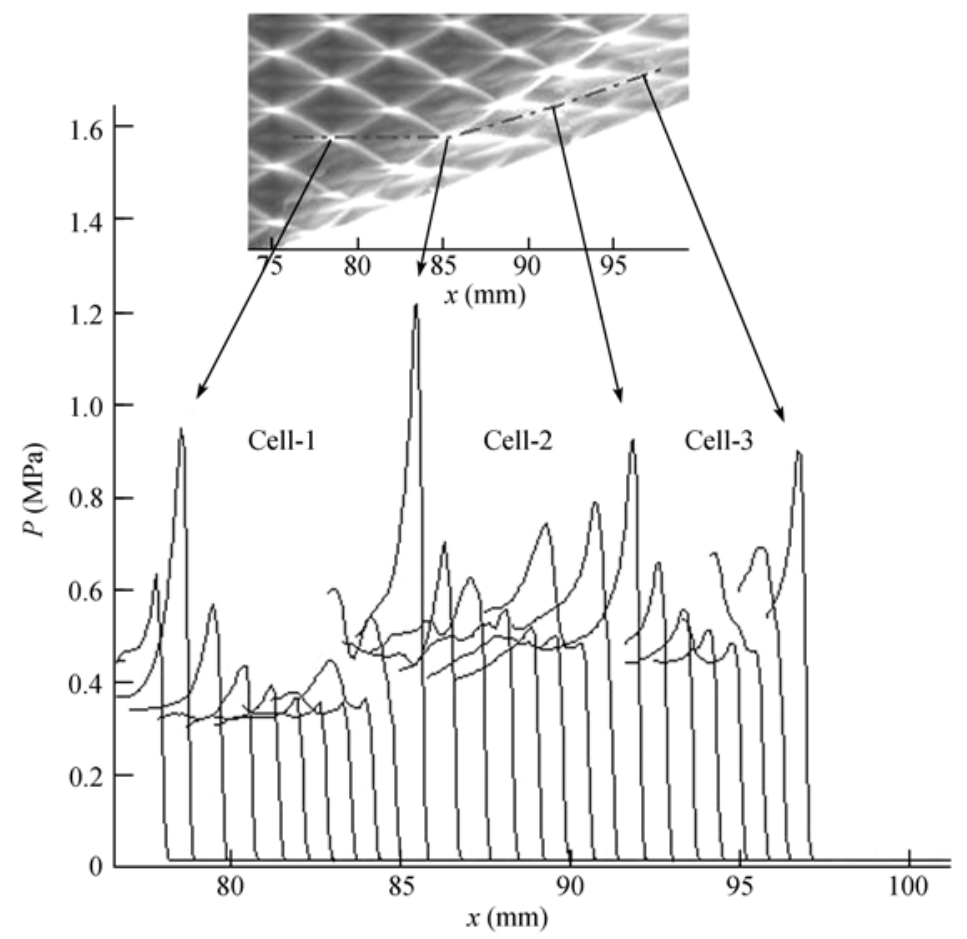

Figure 5 Detonation front pressures recorded along the center line of three cellular cells. 
The post-shock pressure profiles along the centerline of Cell-1 demonstrated that the post-shock pressure decreases first, and then increases to the second peak value. The pressure change reaches $60 \%$ of the first peak pressure. The second pressure peak on the main triple point trajectory is higher than the first one due to the collision between transverse waves and the strong reflected shock. The third and the fourth pressure peaks have almost the same value, and the variation trend of the post-pressure distributions is similar in Cell-2 and Cell-3. This indicates that the Mach stem detonation is stable since Cell-2 and Cell-3 are located in the disturbed region. Moreover, the pressure profiles along the centerline of Cell-2 and Cell-3 are higher than that in Cell-1, which presents a good illustration that the Mach stem detonation is an overdriven detonation compared with the incident detonation. However, for such an overdriven detonation, the third and the fourth peak pressure values are lower than the first one, and this is contrary to the conclusion of the overdriven detonation and needs to be cleared out.

In order to explore this problem, at the time instant when the third peak pressure is generated, the mass fractions of pressure, temperature, $\mathrm{OH}$ and $\mathrm{H}$ were plotted out in Figure 6. By comparing the region behind the incident detonation with that behind the Mach Stem detonation, one can see that
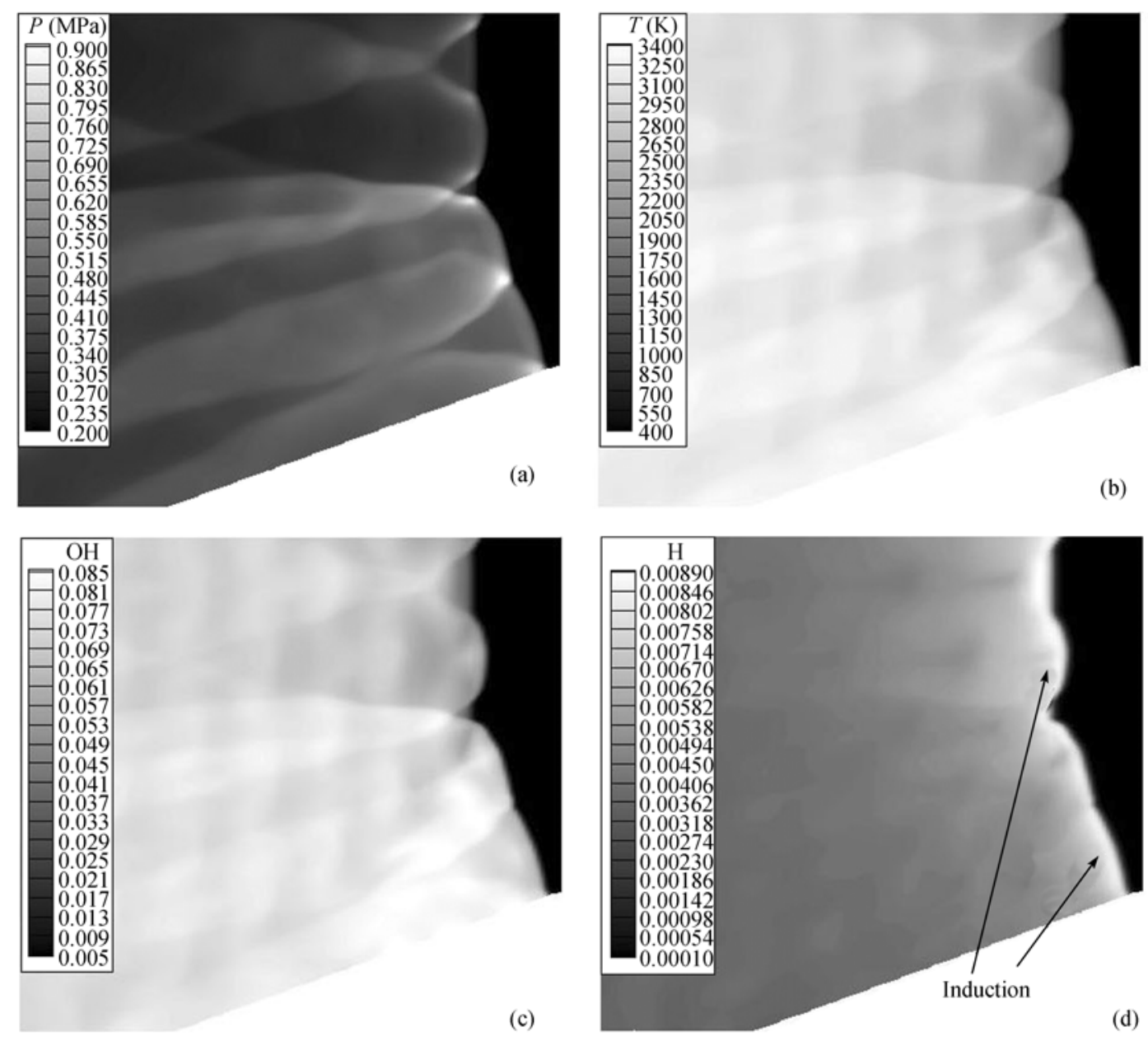

Figure 6 Distributions of pressure, temperature, $\mathrm{OH}$ and $\mathrm{H}$ mass fractions at the time when the third peak pressure is generated due to triple-point collisions. 
the transverse waves behind the Mach stem are re-enhanced due to shock reflection. Because of the higher pressure and temperature behind the Mach stem, as shown in Figure 6(a) and (b), more $\mathrm{OH}$ was generated, as shown in Figure 6(c). This indicates that the chemical reaction in this region is in the more intensive exothermic stage. The chemical induction zone can be approximately determined by $\mathrm{H}$ mass fraction qualitatively as shown in Figure 6(d), where there is the higher production rate of $\mathrm{H}$ mass fraction behind the Mach stem than that behind the incident detonation, which indicates the higher chemical reaction rate and temperature, and the stronger exothermic reaction, which lead to a shorter length of chemical induction zone behind the Mach stem. Thus, the lower peak pressure generated from the triple point collision along the Mach stem is because of the combustible constituents decreasing and chemically-released energy reducing. The length scale of the chemical induction zone is shown in Figure 7 quantitatively, and it is considered that the $\mathrm{H}$ distribution length is proportional to the reaction induction scale and indicates the gas dissolution. It can be seen that the $0.25 \mathrm{~mm}$ dissolution length behind the Mach stem is obviously shorter than the $0.36 \mathrm{~mm}$ length behind the incident detonation. The physical phenomenon can be explained as that the detonation reflection increases gas temperature and strengthens the transverse waves. As a result, the speed increase of transverse waves shortens the length scale of detonation cells. Moreover, the intensive chemical reaction behind the Mach stem reduces the reactants involved in the triple point collision, which leads to the less intensive explosion and the lower peak pressure. For the detonation propagating in the channel with area-converging cross section, the flow direction changing from the wedge behind the detonation provides with a continuous flow compression that drives an overdriven detonation propagating in a steady stage.

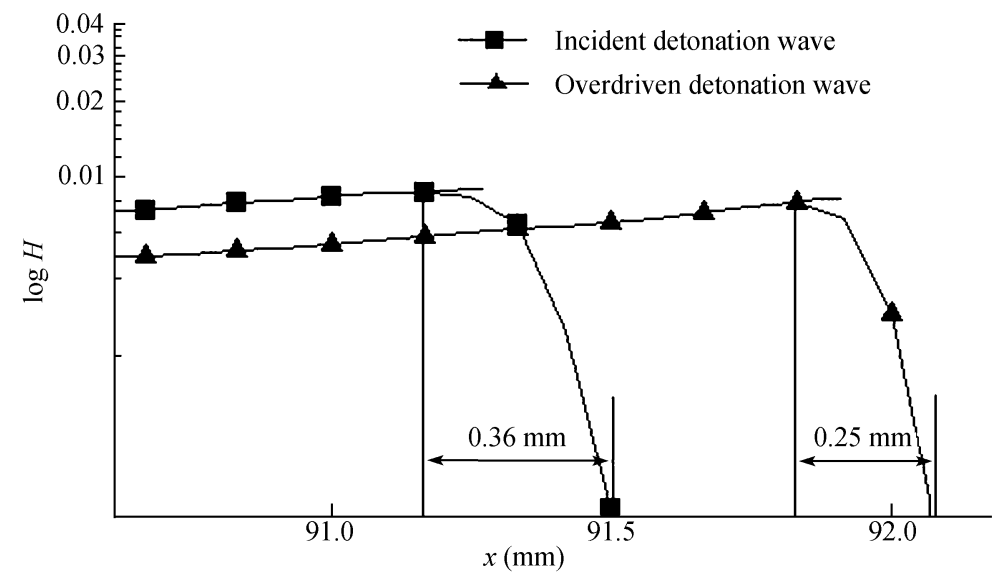

Figure 7 Reaction lengths of the incident and overdriven detonations, indicated by the H distribution.

\subsection{Detonation cells in an expanding channel}

Cellular detonation cells pattern in an expanding channel with a $20^{\circ}$ divergent wall is shown in Figure 8. Obviously, the cellular detonation front disassembles into two parts by a group of the malformed cells. The left-upper cells called the "normal cells" have never been affected by flow expansion. While the right-low cells called the "diffraction cells" become larger and imply an under-driven detonation. The good uniformity of the diffraction cells near the divergent wall indicates that the deflected detonation is steady. 


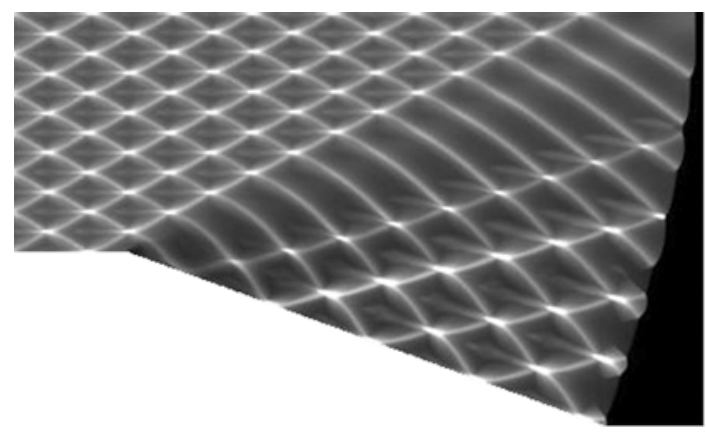

Figure 8 Cellular detonation cells pattern of detonation propagation in a planar channel with a $20^{\circ}$ expanding angle.

Figure 9 shows the successive post-shock pressure profiles along the centerline (marked by the dot-dash line in the subfigure) of four typical cells (labeled as Cell-4, Cell-5, Cell-6, and Cell-7). Five peak pressures are observable at the endpoint of the four cells. Cell-4 still locates in the region swept only by the incident detonation, and the first and the second peak pressures indicate the triple point collision along the incident detonation before its diffraction. Because of the flow expansion from the detonation diffraction, the pressure profile in Cell-5 decreases first, and then increases to the third peak pressure that is lower than the first one, which shows the effect of the flow expansion on thermodynamic parameters. Cell-6 and Cell-7 locate in the detonation diffraction region and the similar trend of the pressure distributions within these two cells indicates that the diffracted detonation becomes steady. The pressure profiles in Cell-6 and Cell-7 appear to be lower than that in Cell-4, which presents a good illustration that the diffracted detonation is under-driven. However, the fourth and fifth pressure peaks look obviously higher than the first and the second ones, which

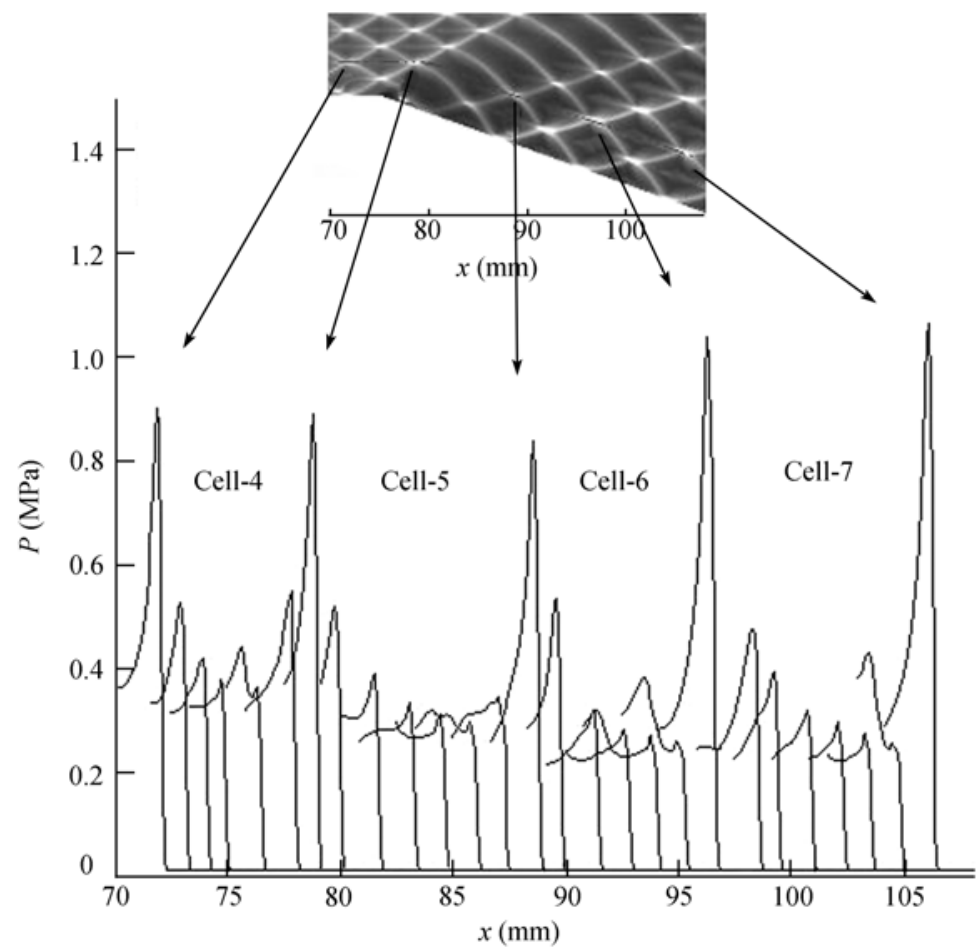

Figure 9 Detonation front pressures recorded along the center line of four cellular cells. 
indicate the more intensive chemical reactions during the triple point collision behind the diffracted detonation.

To investigate further the phenomenon, the mass fraction of pressure, temperature and $\mathrm{H}$ distributions at the time instant when the fourth peak pressure was generated are shown in Figure 10. From Figure 10(a) and (b), it can be seen that the pressure and temperature are lower in most of the expansion region. The relatively low generation rate of $\mathrm{H}$ is shown in Figure 10(c), which indicates the lower chemical reaction rate and the weaker exothermic reaction behind the diffracted detonation. Therefore, more reactants are involved in the triple point collisions and the higher peak pressures were generated.
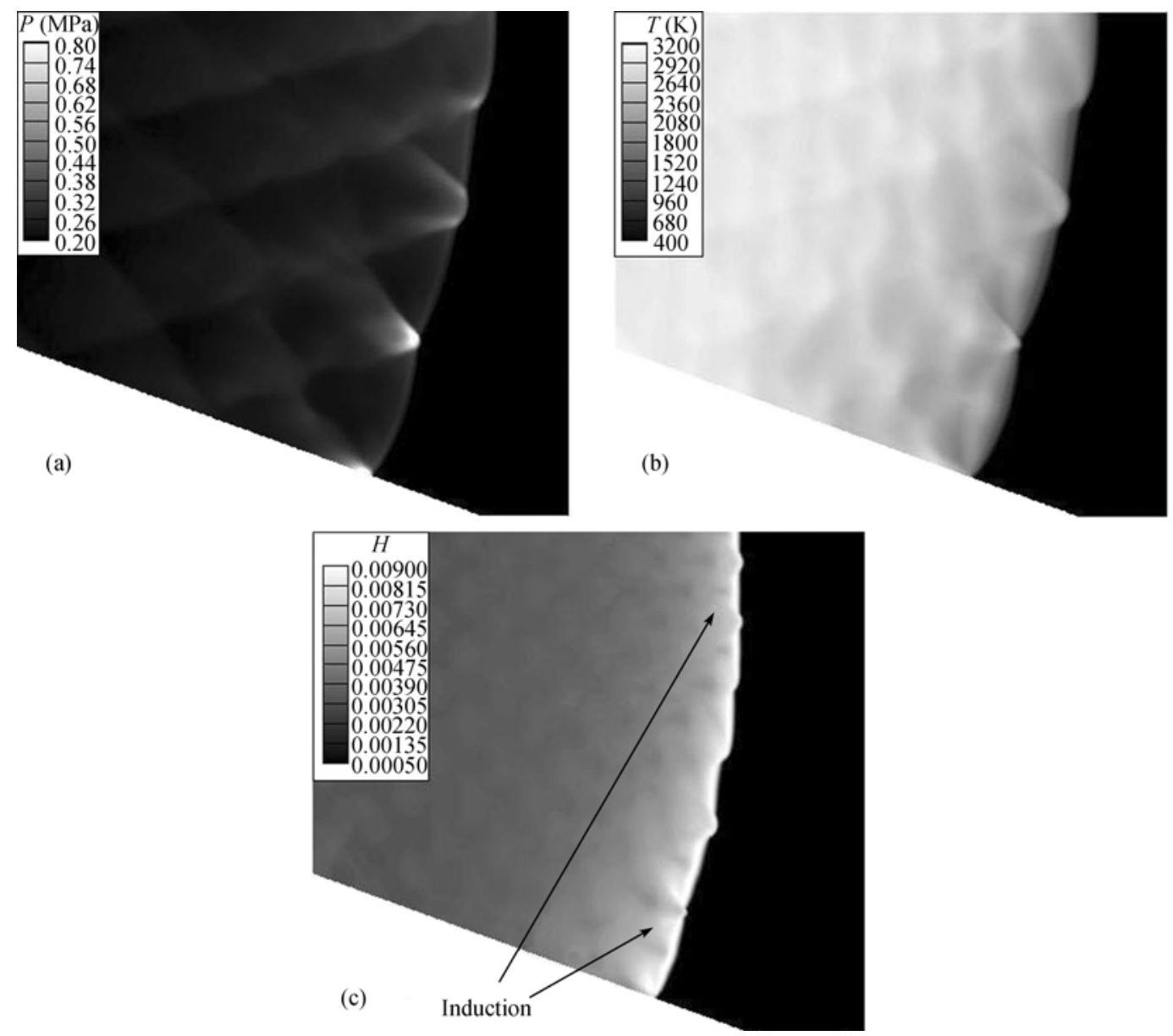

Figure 10 Distributions of pressure, temperature and $\mathrm{H}$ mass fraction at the time when the third peak pressure is generated due to triple-point collisions.

The numerical results show that the $0.46 \mathrm{~mm}$ dissolution reaction length of $\mathrm{H}$ behind the diffracted detonation is longer than the $0.36 \mathrm{~mm}$ reaction length behind the incident detonation. The lower temperature in the expansion region reduces the transverse wave speed, which results in a larger cell scale. This conclusion is in good agreement with Desbordes prediction and demonstrates that the Desbordes equation is not only applied to the overdriven detonation but also to the under-driven detonation. For the detonation propagating in the channel with area-expanding cross 
section, the flow expansion behind the detonation provides with a continuous force that drives an under-driven detonation propagating in a quasi-steady stage.

\section{Conclusion}

By examining the cellular detonation cell and the chemical induction zone length scales, two kinds of the abnormal detonation waves were identified and the mechanism of the cellular cell evolution related to flow compression and expansion was explored. Some conclusions are summarized as follows.

(1) When the cellular detonation propagates in a channel with area-changing cross section, the quasi-steady overdriven and under-driven detonation waves can be generated by the continuous support of the flow expansion and compression. Similar to the one-dimensional CJ detonation, these two-dimensional overdriven and under-driven detonation waves are also self-sustained.

(2) Compared with the normal detonation, the overdriven detonation in a converging channel will induce the higher post-shock temperature and pressure, the stronger chemical reactions, the smaller detonation cell and the lower pressure peaks resulting from triple point collisions; while the under-driven detonation in an expanding channel will generate the lower post-shock temperature and pressure, the less intensive chemical reactions, the bigger detonation cell and the higher peak pressures of triple point collisions. These phenomena can be attributed to the coupling of the leading shock, transverse waves and chemical reaction zones.

(3) The relation between the cellular detonation cell scale and the chemical reaction length scale given by Desbordes is not only applied to the overdriven detonation but also to the under-driven one.

1 Fickett W, Davis W C. Detonation. Los Angeles: University of California Press, 1979

2 Lee J H S. Dynamic parameters of gaseous detonation. Ann Rev Fluid Mech, 1984, 16: 311-336[DOI]

3 Shepherd J E, Pintgen F, Austin J M, et al. The structure of the detonation front in gases. AIAA 40th Aerospace Sciences Meeting. Reno: AIAA, 2002. AIAA-2002-0773

4 Jones D A, Kemister G, Tonello N A, et al. Numerical simulation of detonation re-ignition in $\mathrm{H}_{2}-\mathrm{O}_{2}$ mixtures in area expansions. Shock Waves, 2000, 10: 33-41[DOI]

5 Guo C M, Zhang D L, Xie W. The Mach reflection of a detonation based on soot track measurements. Combust Flame, 2001, 127: 2051-2058[DOI]

6 Thomas G O, Williams R L. Detonation interaction with wedges and bends. Shock Waves, 2002, 11: 481-492[DOI]

7 Jiang Z, Falcovitz J, akayama K T. Numerical simulation of detonation in converging chambers. JSME Int J Ser B, 1997, 40(3): $422-431$

8 Jiang Z, Zhao W, Wang C. Forward running detonation drivers for high-enthalpy shock tunnels. AIAA J, 2002, 40(10): 2009-2016

9 Jiang Z, Takayama K, Chen Y S. Dispersion conditions for non-oscillatory shock capturing schemes and its applications. Comput Fluid Dyn J, 1995, 2(4): 137-150

10 Jiang Z. On the dispersion-control principles for non-oscillatory shock capturing schemes. Acta Mech Sin, 2004, 20(1): $1-15$

11 Teng H H, Jiang Z L. Gasdynamic characteristics of toroidal shock and detonation wave converging. Sci China Ser G-Phys Astron, 2005, 48(6): 739-749[DOI]

12 Desbordes D. Transmission of overdriven plane detonations: Critical diameter as a function of cell regularity and size. AIAA Prog Astronaut Aeronaut, 1988, 114: 170-186 